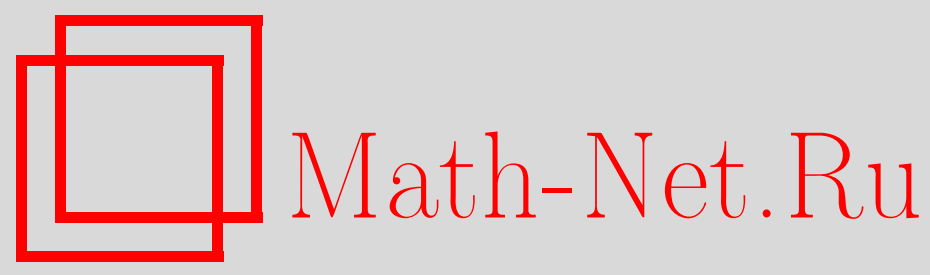

А. С. Олийнык, О свободных полугруппах автоматных преобразований, Матем. заметки, 1998, том 63, выпуск 2, $248-$ 259

DOI: https://doi.org/10.4213/mzm1271

Использование Общероссийского математического портала Math-Net.Ru подразумевает, что вы прочитали и согласны с пользовательским соглашением http://www . mathnet.ru/rus/agreement

Параметры загрузки:

IP : 18.234 .156 .22

26 апреля 2023 г., 17:19:41

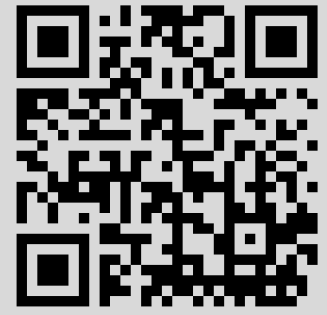




\title{
О СВОБОДНЫХ ПОЛУГРУППАХ АВТОМАТНЫХ ПРЕОБРАЗОВАНИЙ
}

\author{
А. С. Олийнык
}

\begin{abstract}
Установлено, что подмножество свободных $k$-порожденных подполугрупп полугруппы всех автоматных преобразований над конечным алфавитом является множеством второй категории (в смысле категорного подхода Бэра) в множестве всех $k$-порожденных подполугрупп. Указана континуальная серия пар автоматных преобразований, порождающих свободную полугруппу ранга 2 ; установлен критерий, когда такая полугруппа является конечно-автоматной.

Библиография: 15 названий.
\end{abstract}

\section{1. Введение}

Автоматные преобразования, полугруппы и групшы автоматных преобразований нашли широкое применение в различных разделах современной алгебры (см., например, обзор [1]). Цельй ряд работ разных авторов посвящен исследованию строения группы автоматных подстановок над конечньм алфавитом. Согласно [2] группа автоматных подстановок над алфавитом $A$ разлагается в сплетение по бесконечной последовательности (в смысле [3]) симметрических групп $S(A)$. Она может быть реализована так же, как группа автоморфизмов дерева, групша изометрий метрического пространства Бэра и др. Это позволяет достаточно подробно изучить ее строение. Аналогично, полугруппа всех автоматных преобразований над алфавитом $A$ разлагается в сплетение по бесконечной последовательности полугрупп (в смысле [4]). Однако, подробное изучение свойств и строения этой полугрупшы не проводилось.

Яркие результаты о группе автоматных подстановок получены в работах С. В. Алёшина [5] и [6]. В частности, в [6] построен пример автоматов, которые задают преобразования, порождающие свободную группу ранга 2 (полное доказательство пока не опубликовано). Следует отметить, что в связи с известной альтернативой Титса [7] задаче исследования множества свободных подгрупп в различных группах преобразований уделялось достаточно внимания. Так, в работах Д. Эпштейна [8], Д. Диксона [9] и М. Бхаттачаржи [10] доказано, что "большинство" конечно порожденных подгрупा соответственно в группах Ли, симметрических группах на бесконечньх множествах и сплетениях по бесконечным последовательностям нетривиальных групп являются свободными. В связи с этими результатами Р.И. Григорчук поставил задачу - развить методику построения свободных подгрупп и свободных полугрупп (конечно-) автоматных преобразований и исследовать свойства множества таких подполугрупп или подгрупп.

Работа выполнена при частичной финансовой поддержке Международной программы поддержки образования в отрасли точных наук (ISSEP), грант GSU 051341. 
В данной работеприводится частичное решение этой задачи. А именно, устанавливается, что подмножество свободных $k$-порожденных подполугрупп полугрупшы всех автоматных преобразований является множеством второй категории (в смысле категорного подхода Бэра) в множестве всех $k$-порожденных подполугруп, т.е. можно говорить, что “большинство" конечно порожденных подполугрупп полугруппы всех автоматных преобразований является свободным.

Указана континуальная серия пар автоматных преобразований, порождающих свободную полугруппу ранга 2 ; установлен критерий, когда такая полугруппа будет конечно-автоматной. В терминах сплетений выделяется специальный класс автоматных преобразований, 2-элементныеподмножества которого могут порождать свободную полугруппу, и приводятся достаточные условия такой порождаемости.

\section{2. Полугруппа автоматных преобразований}

2.1. Пусть $A$ - алфавит, $|A|=n \geqslant 2, A^{*}$ и $A^{\infty}$ - множества всех соответственно конечных и бесконечных слов над $A$. Для любого инициального автомата $\mathscr{V}=(A, Q, A$, $\left.\varphi, \psi, q_{0}\right)$ с входным и выходным алфавитом $A$, алфавитом состояний $Q$, функцией переходов $\varphi$, функцией выходов $\psi$ и начальньм состоянием $q_{0}$ определено преобразование множества $A^{*}$, задаваемое этим автоматом.

А именно, если $\Lambda$-пустое слово из $A^{*}, \alpha \in A^{*}, a \in A$, то, определив для произвольного $q \in Q \quad \bar{\varphi}(q, \Lambda)=q, \bar{\varphi}(q, \alpha a)=\varphi(\bar{\varphi}(q, \alpha), a)$, имеем $\Lambda^{\mathscr{V}}=\Lambda,(\alpha a)^{\mathscr{V}}=\alpha^{\mathscr{V}} \psi\left(\bar{\varphi}\left(q_{0}, \alpha\right), a\right)$, т.е. если $\alpha=a_{1} a_{2} \cdots a_{m}, a_{i} \in A, 1 \leqslant i \leqslant m$, то

$$
\alpha^{\mathscr{V}}=\psi\left(q_{0}, a_{1}\right) \psi\left(\bar{\varphi}\left(q_{0}, a_{1}\right), a_{2}\right) \cdots \psi\left(\bar{\varphi}\left(q_{0}, a_{1} \cdots a_{m-1}\right), a_{m}\right) .
$$

ОПрЕДЕЛЕниЕ 1 . Преобразование множества $A^{*}$, задаваемое некоторым автоматом $\mathcal{V}$, называется автоматным преобразованием над алфавитом $A$.

ПрЕДЛОЖЕНИЕ 1 [11]. Преобразование $f: A^{*} \rightarrow A^{*}$ будет автоматным (над алфавитом $A$ ) тогда и только тогда, когда выполняются следующие условия:

1) из того что слово $\alpha \in A^{*}$ имеет длину $m$, следует, что и слово $f(\alpha)$ имеет длину $m$;

2) если для слов $\alpha$ и $\beta$ из $A^{*}$ вида $\alpha=a_{1} a_{2} \cdots a_{m}, \beta=b_{1} b_{2} \cdots b_{m}, a_{i}, b_{i} \in A$, $1 \leqslant i \leqslant m$, имеют место равенства $a_{i}=b_{i}, 1 \leqslant i \leqslant s$, при некотором $s$, $1 \leqslant s \leqslant m$, то для их образов $f(\alpha)=a_{1}^{\prime} a_{2}^{\prime} \cdots a_{m}^{\prime} u f(\beta)=b_{1}^{\prime} b_{2}^{\prime} \cdots b_{m}^{\prime}$ выполнено $a_{i}^{\prime}=b_{i}^{\prime}, 1 \leqslant i \leqslant s$.

Если определено автоматное преобразование $f$ множества $A^{*}$, то определено и преобразование $\bar{f}$ множества $A^{\infty}$, которое также будем называть автоматньц.

А именно, если $\bar{\alpha} \in A^{\infty}, \bar{\alpha}=a_{1} a_{2} \cdots a_{m} \cdots$, то при любом $i \geqslant 1$ для слова $a_{1} a_{2} \cdots a_{i}$ из $A^{*}$ определено $f\left(a_{1} a_{2} \cdots a_{i}\right)$, причем по условию 2$)$ предложения 1 слово $f\left(a_{1} a_{2} \cdots a_{i}\right)$ является началом слова $f\left(a_{1} a_{2} \cdots a_{i} a_{i+1}\right)$ при любом $i \geqslant 1$. Следовательно, $\bar{f}(\bar{\alpha})$ может быть определено как бесконечное слово над алфавитом $A$, для которого началом длины $i$ является $f\left(a_{1} a_{2} \cdots a_{i}\right)$.

Пусть $f_{1}$ и $f_{2}$ - автоматные преобразования множеств $A^{*}$ и $A^{\infty}$, задаваемые соответственно автоматами $\mathscr{V}_{1}=\left(A, Q_{1}, A, \varphi_{1}, \psi_{1}, q_{01}\right)$ и $\mathscr{V}_{2}=\left(A, Q_{2}, A, \varphi_{2}, \psi_{2}, q_{02}\right)$. Тогда произведение этих преобразований $f_{1} f_{2}$, определяемое как суперпозиция $f_{2}\left(f_{1}\right)$, также является автоматным и может быть задано автоматом $\mathscr{V}=\left(A, Q, A, \varphi, \psi, q_{0}\right)$, где $Q=Q_{1} \times Q_{2}, q_{0}=\left(q_{01}, q_{02}\right)$,

$$
\begin{gathered}
\varphi\left(\left(q_{1}, q_{2}\right), a\right)=\left(\varphi_{1}\left(q_{1}, a\right), \varphi_{2}\left(q_{2}, \psi_{1}\left(q_{1}, a\right)\right)\right), \\
\psi\left(\left(q_{1}, q_{2}\right), a\right)=\psi_{2}\left(q_{2}, \psi_{1}\left(q_{1}, a\right)\right),
\end{gathered} \quad\left(q_{1}, q_{2}\right) \in Q, \quad a \in A .
$$


Таким образом, все автоматные преобразования над алфавитом $A$ образуют полугруппу с единищей (тождественное преобразование), которую будем обозначать $A T_{n}$. Эта полугруппа действует как на множестве $A^{*}$, так и на $A^{\infty}$, т.е. можно рассматривать две полугруппы преобразований: $\left(A T_{n}, A^{*}\right)$ и $\left(A T_{n}, A^{\infty}\right)$.

2.2. Пусть $\left(S_{i}, M_{i}\right)$ - бесконечная последовательность полугрупп $S_{i}$, действующих на множествах $M_{i}, i \in \mathbb{N}$.

ОПРЕДЕЛЕниЕ 2. Сnлетением по бесконечной последовательности полугрупп преобразований $\left(S_{i}, M_{i}\right), i \in \mathbb{N}$, назьвается полугруппа $S$ всех преобразований $s$ множества $M=\prod_{i \in \mathbb{N}} M_{i}$, удовлетворяющих следуюшим условиям:

1) для любой последовательности $y=\left(y_{1}, y_{2}, \ldots\right)$ значение $k$-й координаты ее образа $y^{s}=\left(y_{1}^{\prime}, y_{2}^{\prime}, \ldots\right)$ зависит лиш от $k$ первых координат $y$;

2) при фиксированных $y_{1}, y_{2}, \ldots, y_{k-1}$ преобразование множества $M_{k}$, определяемое равенством $\widehat{s}_{k}\left(y_{k}\right)=y_{k}^{\prime}$, содержится в $S_{n}$.

Мы будем обозначать сплетение по бесконечной последовательности полугрупп $\left(S_{i}, M_{i}\right)$, $i \in \mathbb{N}$, символом $\iota_{i \in \mathbb{N}} S_{i}$.

Символом $\bar{x}_{k}$ обозначим начало длины $k$ последовательности $\bar{x}=\left(x_{1}, x_{2}, \ldots\right)$ из $A^{\infty}$, т.е. $\bar{x}_{k}=\left(x_{1}, x_{2}, \ldots, x_{k-1}\right)$. Элементы полугруппы $\ell_{i \in N} S_{i}$ можно задавать бесконечными последовательностями, назьваемыми таблииами, которые имеют вид

$$
u=\left[f_{1}, f_{2}\left(x_{1}\right), \ldots, f_{k}\left(\bar{x}_{k-1}\right), \ldots\right]
$$

где $f_{1} \in S_{1}, f_{k}\left(\bar{x}_{k-1}\right)=f_{k}\left(x_{1}, \ldots, x_{k-1}\right)$-произвольное отображение из $\prod_{i=1}^{k-1} M_{i}$ в полугруппу $S_{k}, k=2,3, \ldots$ При этом $k$-ю координату таблицы $u$ будем обозначать $[u]_{k}$.

Сплетения по бесконечньм последовательностям полугрупп являются частным случаем более общей конструкции сплетений по бесконечньм частично упорядоченньм множествам, которая была предложена вначале для групп [12], а затем распространена на полугруппы [4].

Отметим некоторые элементарные свойства этой конструкции.

С каждой последовательностью полугрупп преобразований $\left(S_{1}, M_{1}\right),\left(S_{2}, M_{2}\right), \ldots$ можно связать новую последовательность, состоящую из $k$-итерированных сплетений этих полугрупп. $k$-итерированное сплетение полугрупп $\left(S_{1}, M_{1}\right), \ldots,\left(S_{k}, M_{k}\right)$ состоит из всевозможных таблиц вида $(1)$, имеющих длину $k:\left[f_{1}, f_{2}\left(x_{1}\right), \ldots, f_{k}\left(\bar{x}_{k-1}\right)\right]$. При любом $k$ определен эпиморфизм $\varphi_{k}: 2_{i=1}^{k+1} S_{i} \rightarrow \imath_{i=1}^{k} S_{i}$, задаваемьй равенством

$$
\varphi_{k}\left(\left[f_{1}, f_{2}\left(x_{1}\right), \ldots, f_{k}\left(\bar{x}_{k-1}\right), f_{k+1}\left(\bar{x}_{k}\right)\right]\right)=\left[f_{1}, f_{2}\left(x_{1}\right), \ldots, f_{k}\left(\bar{x}_{k-1}\right)\right]
$$

Тем самым, задается обратный спектр полугрупп преобразований $\left(l_{i=1}^{k+1} S_{i}, \varphi_{k}\right) k_{k \in \mathbb{N}}$.

ПРЕДЛОЖЕНИЕ 2. Предельная полугруппа обратного спектра $\left(l_{i=1}^{k+1} S_{i}, \varphi_{k}\right)_{k \in \mathbb{N}}$ изоморфна, как полугруппа преобразований, сплетению $\imath_{i \in \mathbb{N}} S_{i}$.

ПРЕДЛОЖЕНИЕ 3. Сплетение по бесконечной последовательности полугрупп преобразований является внутренне ассочиативной конструкиией, т.е. для любой последовательности $\left(S_{i}, M_{i}\right), i \in \mathbb{N}$, при произвольном $k \in \mathbb{N}$ сплетения $\left(l_{i=1}^{k} S_{i}\right) \curlywedge\left(\imath_{i=k+1}^{\infty} S_{i}\right)$ u $\iota_{i \in \mathbb{N}} S_{i}$ подобньl, как полугруппь преобразований. 
ПРЕДЛОЖЕНИЕ 4. ДЛя любого въбора подполугрупп $P_{i}$ из $S_{i}$ сплетение $l_{i \in \mathbb{N}} S_{i}$ содержит подполугруппу $\imath_{i \in \mathbb{N}} P_{i}$.

Если при каком-то $k \in \mathbb{N} \quad S_{k}=\bigcap_{\lambda \in \Lambda} S_{k \lambda}$ u $S(\lambda)$ - сплетение по последовательности $\left(S_{1}, M_{1}\right), \ldots,\left(S_{k-1}, M_{k-1}\right),\left(S_{k \lambda}, M_{k}\right),\left(S_{k+1}, M_{k+1}\right), \ldots$, причем все полугруппы $S_{i}(i \in \mathbb{N}), S_{k \lambda}(\lambda \in \Lambda)$ с единицей, mо $l_{i \in \mathbb{N}} S_{i}=\bigcap_{\lambda \in \Lambda} S(\lambda)$.

Если $\left|M_{i}\right|<\infty$ nрu $i \leqslant k-1$ u $S_{k}=\left\langle S_{k \lambda}, \lambda \in \Lambda\right\rangle$, mo $_{i \in N} S_{i}=\left\langle S_{k \lambda}, \lambda \in \Lambda\right\rangle$.

ПредЛОЖенИЕ 5. Полугруппа преобразований $\left(l_{i \in \mathbb{N}} S_{i}, \prod_{i \in \mathbb{N}} M_{i}\right)$ транзитивна в том и только том случае, когда каждая из полугрупп $S_{i}$ транзитивно действует на множестве $M_{i}, i \in \mathbb{N}$.

Доказательства этих предложений вполне аналогичны доказательствам соответствуюших утверждений для сплетений групп, поэтому мы их опускаем.

2.3. ПредЛОЖЕНИЕ 6. Полугруппа $\left(A T_{n}, A^{\infty}\right)$ изоморфна, как полугруппа преобразований, сплетению по бесконечной последовательности симметрических полугрупп $T_{n}$ степени $n$ :

$$
\left(A T_{n}, A^{\infty}\right) \simeq\left(l_{i \in \mathbb{N}} T_{n}^{(i)}, \prod_{i \in \mathbb{N}} A\right)
$$

əде $T_{n}^{(i)}=T_{n}, i \in \mathbb{N}$.

ДокАЗАТЕльСтво. Так как имеет место естественное биективное соответствие между множеством $A^{\infty}$ всех бесконечных слов над алфавитом $A$ и бесконечной декартовой степенью множества $A$, можно считать, что сплетение $\imath_{i \in \mathbb{N}} T_{n}^{(i)}$ действует на множестве $A^{\infty}$. Сплетение полных симметрических полугрупп $T_{n}$ состоит из преобразований, удовлетворяюших лишь условию 1) определения 2. Но это условие выполняется для автоматных над алфавитом $A$ преобразований и только для них, откуда и получаем требуемое утверждение.

Согласно предложению 6 автоматные преобразования над $A$ можно задавать таблицами вида

$$
u=\left[f_{1}, f_{2}\left(x_{1}\right), \ldots, f_{k}\left(\bar{x}_{k-1}\right), \ldots\right], \quad \text { где } f_{1} \in T_{n}, f_{k}: A^{k-1} \rightarrow T_{n} .
$$

Координаты произведения $u v$ таблиц

$$
u=\left[f_{1}, f_{2}\left(x_{1}\right), \ldots, f_{k}\left(\bar{x}_{k-1}\right), \ldots\right] \in A T_{n}, \quad v=\left[g_{1}, g_{2}\left(x_{1}\right), \ldots, g_{k}\left(\bar{x}_{k-1}\right), \ldots\right] \in A T_{n}
$$

выражаются через координаты $u$ и $v$ равенством

$$
[u v]_{k}=f_{k}\left(\bar{x}_{k-1}\right) g_{k}\left(x_{1}^{f_{1}}, x_{2}^{f_{2}\left(x_{1}\right)}, \ldots, x_{k-1}^{f_{k-1}\left(\bar{x}_{k-2}\right)}\right) .
$$

Таблица $\varepsilon=[\varepsilon, \varepsilon, \ldots]$, где $\varepsilon$ - тождественное преобразование из $T_{n}$, является единицей в $A T_{n}$.

Далее мы часто будем отождествлять автоматное преобразование с таблицей, которая его определяет. По таблице можно также строить информационное дерево преобразования (см. [11]) и автомат, его задаюший. 


\section{3. Большинство подполугрупп в $A T_{n}$ свободны}

3.1. ОПРЕДЕЛЕНИЕ 3 . Таблицу $u \in A T_{n}$ вида

$$
u=\left[f_{1}, f_{2}\left(x_{1}\right), \ldots, f_{m}\left(\bar{x}_{m-1}\right), \varepsilon, \ldots, \varepsilon, \ldots\right], \quad f_{m}\left(\bar{x}_{m-1}\right) \neq \varepsilon,
$$

будем назьвать короткой длины $m$.

Продолжением короткой таблицы (3) назовем любую таблицу $v \in A T_{n}$ вида

$$
v=\left[f_{1}, f_{2}\left(x_{1}\right), \ldots, f_{m}\left(\bar{x}_{m-1}\right), g\left(\bar{x}_{m}\right), \ldots\right] .
$$

Определим на полугруппе $A T_{n}$ метрику $d$, полагая для любых $u, v \in A T_{n}$

$$
d(u, v)= \begin{cases}0, & \text { если } u=v, \\ 2^{-m(u, v)} & \text { в противном случае, }\end{cases}
$$

где $m(u, v)$ - наименьший номер такой, что $[u]_{m(u, v)+1} \neq[v]_{m(u, v)+1}$.

Очевидно, $\left(A T_{n}, d\right)$ - метрическое пространство с неархимедовой метрикой. Открытый шар этого пространства с центром в точке $u$ радиуса $2^{-m}, m \geqslant 0$, будем обозначать через $B(u, m)$.

Тогда таблица $v$ будет продолжением короткой таблицы $u$ длины $m$, если $v \in B(u, m)$.

Лемма 1. Полугрупповая операция на $A T_{n}$ непрерьвна в метрике (4), причем метрическое пространство $\left(A T_{n}, d\right)$ является полным.

ДокаЗАтельСтво. Пусть $u, v \in A T_{n}, m \in \mathbb{N}$. Если для некоторых $u_{1}, v_{1} \in A T_{n}$ первые $m$ координат $u$ и $u_{1}$, а также $v$ и $v_{1}$, совпадают, то совпадают и первые $m$ координат $u v$ и $u_{1} v_{1}$. Отсюда следует непрерьвность полугрупповой операции в метрике (4).

Пусть $\left\{u_{i}: i \in \mathbb{N}\right\}$ - произвольная фундаментальная последовательность точек метрического пространства $\left(A T_{n}, d\right)$. Это означает, что для любого $m \in \mathbb{N}$ существует короткая таблица $v_{m}$ длины $k_{m} \geqslant m$ такая, что лишь конечное число членов последовательности $\left\{u_{i}\right\}$ не является ее продолжением. При этом $v_{m}$ будет продолжением $v_{m^{\prime}}$, когда $m \geqslant m^{\prime}$. Для последовательности коротких таблиц $\left\{v_{m}: m \in \mathbb{N}\right\}$ существует единственная таблища $u$, являющаяся продолжением $v_{m}$ при любом $m \in \mathbb{N}$. Она определяется покоординатно равенствами $[u]_{j}=\left[v_{m}\right]_{j}$, где $m$-наименьшее число такое, что $k_{m} \geqslant j$. Тогда $\lim _{i \rightarrow \infty} u_{i}=u$, т.е. для последовательности $\left\{u_{i}: i \in \mathbb{N}\right\}$ существует предел. Так как фундаментальная последовательность $\left\{u_{i}: i \in \mathbb{N}\right\}$ произвольна, это означает, что пространство $\left(A T_{n}, d\right)$ полно. Лемма доказана.

Зафиксируем некоторое $k$ и рассмотрим $A T_{n}^{k}$. Распространим теперь метрику $d$ на множество $A T_{n}^{k}$, полагая

$$
d(u, v)=\max \left\{d\left(u_{i}, v_{i}\right): 1 \leqslant i \leqslant k\right\},
$$

где $u=\left(u_{1}, \ldots, u_{k}\right), v=\left(v_{1}, \ldots, v_{k}\right) \in A T_{n}^{k}$. По лемме $1\left(A T_{n}^{k}, d\right)$-полное метрическое пространство. Открытый шар этого пространства с центром в точке $u$ радиуса $2^{-m}$ будем, как и прежде, обозначать $B(u, m)$.

Напомним, что подмножество метрического пространства называется нигде не nлoтным, если его дополнение содержит открытое плотное подмножество. Объединение счетного числа ниг де неплотных множеств назьвается тощим (или множеством первой категории). Теорема Бэра о категории утверждает, что в полном метрическом пространстве дополнение тощего множества плотно. В частности, пространство не может быть представлено в виде объединения счетного семейства тощих множеств (см., например, [13]).

Для кортежа $u=\left(u_{1}, \ldots, u_{k}\right) \in A T_{n}^{k}$ символом $\langle u\rangle$ будем обозначать подполугруппу $A T_{n}$, порожденную его компонентами. 
3.2. Пусть $X=\left(X_{1}, \ldots, X_{k}\right)$ - образуюшие свободной полугрупшы $\operatorname{FS}(X)$ ранга $k$. Если $w=w(X)$ - некоторое слово из этой полугруппы, то $w(u)$ - значение этого слова на кортеже $u$.

Рассмотрим следующие подмножества из $A T_{n}^{k}$ :

$$
F=\{u:\langle u\rangle \simeq \mathrm{FS}(X)\}, \quad F\left(w_{1}, w_{2}\right)=\left\{u: w_{1}(u) \neq w_{2}(u)\right\}
$$

где $w_{1}, w_{2}$ - некоторые слова из $\mathrm{FS}(X)$.

Лемма 2. $F=\bigcap_{w_{1} \neq w_{2}} F\left(w_{1}, w_{2}\right)$.

ДокАЗАТЕЛЬСтво. Кортеж $u$ не содержится в $F$ тогда и только тогда, когда между его компонентами имеются нетривиальные соотношения. Если $w_{1}(u)=w_{2}(u)$ одно из них, то $u$ не содержится в $F\left(w_{1}, w_{2}\right)$, а следовательно, и в $F=\bigcap_{w_{1} \neq w_{2}} F\left(w_{1}, w_{2}\right)$. Отсюда следует требуемое равенство.

Лемма 3. Для любых $w_{1}, w_{2} \in \mathrm{FS}(X), w_{1} \neq w_{2}$, подмнохсество $F\left(w_{1}, w_{2}\right)$ открыто.

ДокАЗАТЕЛЬСТвО. Отображения $u \mapsto w_{1}(u)$ и $u \mapsto w_{2}(u)$ непрерьвны в силу леммы 1. Поэтому $F\left(w_{1}, w_{2}\right)$ является дополнением множества $A T_{n}^{k} \backslash F\left(w_{1}, w_{2}\right)$, которое замкнуто вследствие непрерьвности этих отображений. Следовательно, само оно открыто, и утверждение леммы доказано.

Лемма 4. Пусть $w_{1}(X)=X_{\alpha_{1}} X_{\alpha_{2}} \cdots X_{\alpha_{l}}, w_{2}(X)=X_{\beta_{1}} X_{\beta_{2}} \cdots X_{\beta_{r}}(l, r \geqslant 0$, $l+r>0)$ - различные слова из $\mathrm{FS}(X)$. Для произвольных коротких таблич $u_{1}, \ldots, u_{k}$ длины $m$ существуют короткие таблицы $v_{1}, \ldots, v_{k}$ длины $m+\max \{l, r\}$, являющиеся их продолжениями, такие, что $w_{1}\left(v_{1}, \ldots, v_{k}\right) \neq w_{2}\left(v_{1}, \ldots, v_{k}\right)$.

ДОкАЗАТЕЛЬСТВО. Если $w_{1}\left(u_{1}, \ldots, u_{k}\right) \neq w_{2}\left(u_{1}, \ldots, u_{k}\right)$, топродолжения $v_{1}, \ldots, v_{k}$ требуемой длины таблиц $u_{1}, \ldots, u_{k}$ могут быть произвольными. Пусть $u_{1}, \ldots, u_{k}-$ короткие таблищы такие, что $w_{1}\left(u_{1}, \ldots, u_{k}\right)=w_{2}\left(u_{1}, \ldots, u_{k}\right)$. Предположим для определенности, что $r \geqslant l$, и обозначим через $s-1$ длину общего начала слов $w_{1}$ и $w_{2}$. Тогда $s$ - наименьшее число такое, что $w_{1}$ и $w_{2}$ различаются в $s$-й позиции, $1 \leqslant s \leqslant l+1$. Пусть $\pi=\left(a^{\prime}, a^{\prime \prime}\right)$ - транспозиция символов $a^{\prime}, a^{\prime \prime} \in A, a^{\prime} \neq a^{\prime \prime}$. Продолжим таблищы $u_{1}, \ldots, u_{k}$ до коротких таблиц $u_{1}^{\prime}, \ldots, u_{k}^{\prime}$ длины $m+s-1$ произвольным образом. Для всех $i, 1 \leqslant i \leqslant k$, координаты их продолжений

$$
v_{i}=\left[\left[u_{i}^{\prime}\right]_{1}, \ldots,\left[u_{i}^{\prime}\right]_{m+s-1}, f_{m+s}^{(i)}\left(\bar{x}_{m+s-1}\right), \ldots, f_{m+r}^{(i)}\left(\bar{x}_{m+r-1}\right), \varepsilon, \ldots\right]
$$

c $(m+s)$-й по $(m+r)$-ю определяем следующими соглашениями. Если вьполнено условие $i=\beta_{s+j}$, то функция $f_{m+s+j}^{(i)}$ принимает значение $\pi$ на всех кортежах вида $\left(a_{1}, \ldots, a_{m+s+t}, a^{\prime \prime}\right)$, где $a_{1}, \ldots, a_{m+s+t}-$ произвольные элементы из $A, t=\max \{j-2$, $-1\}$, и значение $\varepsilon$ на всех остальных кортежах; если же $i \neq \beta_{s+j}$, то $f_{m+s+j}^{(i)}$ на всех кортежах принимает единичное значение. Так определенные таблицы $v_{1}, \ldots, v_{k}$ являются короткими длины $m+r$ и продолжениями $u_{1}, \ldots, u_{k}$ соответственно.

Убедимся, что они являются искомьпи, т.е. вьполнено $w_{1}\left(v_{1}, \ldots, v_{k}\right) \neq w_{2}\left(v_{1}, \ldots, v_{k}\right)$. Достаточно проверить, что таблицы $w_{1}\left(v_{1}, \ldots, v_{k}\right)$ и $w_{2}\left(v_{1}, \ldots, v_{k}\right)$ по-разному действуют хотя бы на одно слово над алфавитом $A$. Покажем, что для слова $\bar{a}^{\prime}=a^{\prime} a^{\prime} \cdots$ имеет место неравенство $\bar{a}^{\prime w_{1}\left(v_{1}, \ldots, v_{k}\right)} \neq \bar{a}^{\prime w_{2}\left(v_{1}, \ldots, v_{k}\right)}$. В самом деле, в слове $\bar{a}^{\prime v_{\alpha_{1}} \cdots v_{\alpha_{s}}}$ $(m+s)$-ю позицию занимает буква $a^{\prime}$, а в слове $\bar{a}^{\prime v_{\beta_{1}} \cdots v_{\beta_{s}}}$ на этом месте стоит $a^{\prime \prime}$. Согласно определению таблиц $v_{1}, \ldots, v_{k}$ отсюда получаем, что на $(m+s+1)$-м месте в 


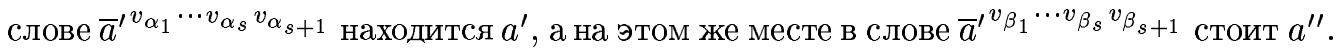
Продолжая те же рассуждения, на $(r-s)$-м шаге получаем, что на $(m+r)$-м месте в словах $\bar{a}^{\prime} v_{\alpha_{1}} \cdots v_{\alpha_{l}}$ и $\bar{a}^{\prime} v_{\beta_{1}} \cdots v_{\beta_{r}}$ находятся $a^{\prime}$ и $a^{\prime \prime}$ соответственно, т.е. требуемое неравенство выполнено. Лемма доказана.

ТЕОрема 1. Подмножество F плотно в $A T_{n}^{k}$.

ДокАЗАтЕЛЬСтво. Пусть $u=\left(u_{1}, \ldots, u_{k}\right) \in A T_{n}^{k}$ и $m \in \mathbb{N}$. Построим $v=\left(v_{1}, \ldots, v_{k}\right)$ $\in B(u, m)$ так, чтобы $v \in F$. Рассмотрим множество $W=\left\{\left(w_{i_{1}}, w_{j_{1}}\right),\left(w_{i_{2}}, w_{j_{2}}\right), \ldots\right\}$ всевозможных пар различных слов из $\mathrm{FS}(X)$. Пусть $l_{t}$ - длина более длинного слова из $t$-й пары. Рассмотрим короткие таблищы $v_{1}^{(0)}, \ldots, v_{k}^{(0)}$ длины $m$ такие, что $v^{(0)}=$ $\left(v_{1}^{(0)}, \ldots, v_{k}^{(0)}\right) \in B(u, m)$. Это значит, что выполняются равенства $\left[v_{i}^{(0)}\right]_{j}=\left[u_{i}\right]_{j}$ для всех $i, j, 1 \leqslant i \leqslant k, 1 \leqslant j \leqslant m$. Для этого набора таблиц и пары $\left(w_{i_{1}}, w_{j_{1}}\right)$ по лемме 4 построим короткие таблицы $v_{1}^{(1)}, \ldots, v_{k}^{(1)}$ длины $m+l_{1}$, которые являются продолжениями таблиц $v_{1}^{(0)}, \ldots, v_{k}^{(0)}$, так, чтобы $w_{i_{1}}\left(v_{1}^{(1)}, \ldots, v_{k}^{(1)}\right) \neq w_{j_{1}}\left(v_{1}^{(1)}, \ldots, v_{k}^{(1)}\right)$. Затем продолжим набор $v_{1}^{(1)}, \ldots, v_{k}^{(1)}$ до набора коротких таблиц $v_{1}^{(2)}, \ldots, v_{k}^{(2)}$ длины $m+l_{1}+l_{2}$ так, чтобы выполнялось неравенство $w_{i_{2}}\left(v_{1}^{(2)}, \ldots, v_{k}^{(2)}\right) \neq w_{j_{2}}\left(v_{1}^{(2)}, \ldots, v_{k}^{(2)}\right)$. Вьполнив эти построения для всех элементов $W$, получим таблищы $v_{1}, \ldots, v_{k}$. Так как два продолжения одного и того же набора коротких таблиц длины $m$ находятся на расстоянии, не превышающем $2^{-m}$, выполнено $v=\left(v_{1}, \ldots, v_{k}\right) \in B(u, m)$. Поскольку неравенства $w_{i_{t}}\left(u_{1}^{\prime}, \ldots, u_{k}^{\prime}\right) \neq w_{j_{t}}\left(u_{1}^{\prime}, \ldots, u_{k}^{\prime}\right)$ сохраняются для любых продолжений $v_{1}^{\prime}, \ldots, v_{k}^{\prime}$ таблиц $u_{1}^{\prime}, \ldots, u_{k}^{\prime}$, то неравенства $w_{i_{t}}(v) \neq w_{j_{t}}(v)$ вьполняются для всех $t \geqslant 1$. Следовательно, $v \in F$. Теорема доказана.

ТЕОрема 2. Подмнохество $A T_{n}^{k} \backslash F$ является тощим в $A T_{n}^{k}$.

ДокАЗАТЕльСтво. Для любых различных слов $w_{1}$ и $w_{2}$ из $\operatorname{FS}(X)$ по лемме 2 имеем $F \subseteq F\left(w_{1}, w_{2}\right)$. Отсюда, учитывая теорему 1 и лемму 3 , получаем, что $F\left(w_{1}, w_{2}\right)$ открыто и плотно в $A T_{n}^{k}$. Следовательно, $A T_{n}^{k} \backslash F\left(w_{1}, w_{2}\right)$ нигде не плотно. Так как

$$
A T_{n}^{k} \backslash F=A T_{n}^{k} \backslash \bigcap_{w_{1} \neq w_{2}} F\left(w_{1}, w_{2}\right)=\bigcup_{w_{1} \neq w_{2}}\left(A T_{n}^{k} \backslash F\left(w_{1}, w_{2}\right)\right),
$$

$A T_{n}^{k} \backslash F$ представимо в виде объединения счетного семейства нигде не плотных подмножеств, т.е. является тощим. Теорема доказана.

\section{4. Конструкция континуального семейства свободных полугрупп в $A T_{p}$}

Предположим, что $|A|=p$ - простое число, и отождествим $A$ с полем $\mathbb{Z}_{p}$ вычетов по модулю $p$. Пусть $C_{p}$ - циклическая группа сдвигов $x \mapsto x+a$ на множестве $\mathbb{Z}_{p}$. Группа $A G_{p}$ всех обратимых преобразований из $A T_{p}$ разлагается в сплетение по бесконечной последовательности симметрических групп над алфавитом $A$ и, следовательно, является проконечной группй. Поэтому можно говорить о ее силовских $p$-подгруппах, которые все сопряжены [14]. Силовская $p$-подгруппа $P_{\infty}$ групшы $A G_{p}$ изоморфна сплетению по бесконечной последовательности циклических групп подстановок $\left(C_{p}, \mathbb{Z}_{p}\right)$ и действует на $\mathbb{Z}_{p}^{\infty}$. Так как $C_{p}$ естественно отождествляется с аддитивной групшой поля $\mathbb{Z}_{p}$, элементы группы $P_{\infty}$ задаются наборами вида $(1)$, координаты которых есть функции над $\mathbb{Z}_{p}$ от соответствующего числа переменных. Следовательно, можно считать, что они являются редуцированными многочленами, т.е. $f_{1} \in \mathbb{Z}_{p}, f_{k}\left(\bar{x}_{k-1}\right) \in$ $\mathbb{Z}_{p}\left[x_{1}, \ldots, x_{k-1}\right] / \mathscr{J}_{k-1}$, где $\mathscr{J}_{k-1}$ - идеал в $\mathbb{Z}_{p}\left[x_{1}, \ldots, x_{k-1}\right]$, порожденный многочленами $x_{1}^{p}-x_{1}, \ldots, x_{k-1}^{p}-x_{k-1}$. 
Поскольку групповая операция в $C_{p}$ записывается аддитивно, действие таблицы $u$ вида (1) на произвольную последовательность $\bar{\alpha}=\left(\alpha_{1}, \alpha_{2}, \alpha_{3}, \ldots\right) \in \mathbb{Z}_{p}^{\infty}$ определяется равенством

$$
\bar{\alpha}^{u}=\left(\alpha_{1}+f_{1}, \alpha_{2}+f_{2}\left(\alpha_{1}\right), \alpha_{3}+f_{3}\left(\alpha_{1}, \alpha_{2}\right), \ldots\right) .
$$

Согласно (5) правило (2) умножения таблиц в этом случае перепишется в виде

$$
[u v]_{k}=f_{k}\left(\bar{x}_{k-1}\right)+g_{k}\left(x_{1}+f_{1}, x_{2}+f_{2}\left(x_{1}\right), \ldots, x_{k-1}+f_{k-1}\left(\bar{x}_{k-2}\right)\right),
$$

а единичной будет таблица $\varepsilon=[0,0, \ldots]$.

Свободные 2-порожденные подполугрупшы в групе $P_{\infty}$ строятся следующим обра3ом.

Пусть сначала $p \neq 2$. Зафиксируем некоторую последовательность $\bar{\alpha}=\left(\alpha_{1}, \alpha_{2}, \ldots\right.$, $\left.\alpha_{k}, \ldots\right) \in \mathbb{Z}_{p}^{\infty}$ и зададим таблицы $u, v \in P_{\infty}$, полагая

$$
\begin{aligned}
& u=\left[0,0, \alpha_{1} x_{2}+x_{1}, \alpha_{2} x_{3}+x_{2}, \ldots, \alpha_{k} x_{k+1}+x_{k}, \ldots\right], \\
& v=\left[0,0, \alpha_{1} x_{2}+x_{1}^{2}, \alpha_{2} x_{3}+x_{2}^{2}, \ldots, \alpha_{k} x_{k+1}+x_{k}^{2}, \ldots\right] .
\end{aligned}
$$

Обозначим через $F(\bar{\alpha})$ полугруппу, порожденную таблищами $u$ и $v$. Условимся считать, что для редуцированного многочлена $f$ запись $f\left(x_{i_{1}}, \ldots, x_{i_{t}}\right)$ означает, что он может явно зависеть лишь от указанных неизвестных, т.е. множество тех неизвестных, от которых он явно зависит, содержится в $\left\{x_{i_{1}}, \ldots, x_{i_{t}}\right\}$.

Нам понадобятся два вспомогательных утверждения о виде многочленов - координат таблиц из $F(\bar{\alpha})$.

Лемма 5. Для любого натурального $m \geqslant 2$ при $k>2 m$ имеют место соотношения

$$
\begin{gathered}
{\left[u^{m}\right]_{k}=x_{k-2 m}+f_{k}^{(m)}\left(x_{k-2 m+1}, \ldots, x_{k-1}\right),} \\
{\left[v^{m}\right]_{k}=\overline{2^{m-1}} x_{k-2 m}^{2} x_{k-2 m+2} \cdots x_{k-4} x_{k-2}+g_{k}^{(m)}\left(x_{k-2 m}, \ldots, x_{k-1}\right),}
\end{gathered}
$$

әде $\overline{2^{m-1}}$ - вычет числа $2^{m-1}$ по модулю $p$, амногочлен $g_{k}^{(m)}$ не содержст одночлена $x_{k-2 m}^{2} x_{k-2 m+2} \cdots x_{k-4} x_{k-2}$.

ДокАЗАТЕЛЬСТво проведем индукцией по числу $m$. Достаточно проверить соотношение (8), соотношение (7) проверяется вполне аналогично.

База индукции - случай $m=2$. Согласно равенству (6) $k$-я координата таблицы $v^{2}$ при $k>4$ имеет вид

$$
\begin{aligned}
& \left(\alpha_{k-2} x_{k-1}+x_{k-2}^{2}\right)+\alpha_{k-2}\left(x_{k-1}+\alpha_{k-3} x_{k-2}+x_{k-3}^{2}\right)+\left(x_{k-2}+\alpha_{k-4} x_{k-3}+x_{k-4}^{2}\right)^{2} \\
& \quad=2 x_{k-4}^{2} x_{k-2}+g_{k}^{(2)}\left(x_{k-4}, \ldots, x_{k-1}\right),
\end{aligned}
$$

т.е. записьвается в требуемом виде.

Шаг индукции. При $k>2 m$ имеем

$$
\left[v^{m}\right]_{k}=\left[v^{m-1}\right]_{k}+\alpha_{k-2}\left(x_{k-1}+\left[v^{m-1}\right]_{k-1}\right)+\left(x_{k-2}+\left[v^{m-1}\right]_{k-2}\right)^{2} .
$$

По предположению индукции многочлены $\left[v^{m-1}\right]_{k}$ и $\alpha_{k-2}\left(x_{k-1}+\left[v^{m-1}\right]_{k-1}\right)$ не зависят от $x_{k-2 m}$, т.е. их сумма является многочленом $\widetilde{f}\left(x_{k-2 m+1}, \ldots, x_{k-1}\right), \mathrm{a}\left[v^{m-1}\right]_{k-2}$ имеет вид

$$
\left[v^{m-1}\right]_{k-2}=\overline{2^{m-2}} x_{k-2 m}^{2} x_{k-2 m+2} \cdots x_{k-4}+g_{k-2}^{(m-1)}\left(x_{k-2 m}, \ldots, x_{k-3}\right) .
$$

Поэтому многочлен $\left(x_{k-2}+\left[v^{m-1}\right]_{k-2}\right)^{2}$ записьвается в виде суммы монома $\overline{2^{m-1}} \times$ $x_{k-2 m}^{2} x_{k-2 m+2} \cdots x_{k-4} x_{k-2}$ и некоторого многочлена $\widetilde{g}\left(x_{k-2 m}, \ldots, x_{k-2}\right)$, его не содержашего. Теперь, полагая $g_{k}^{(m)}=\widetilde{f}+\widetilde{g}$, получаем соотношение (8). 
Лемма 6. Пусть $w\left(X_{1}, X_{2}\right)$ - произвольное нетривиальное слово из свободной полугруппь $\mathrm{FS}\left(X_{1}, X_{2}\right), m$ - его длина. Тогда при $k>2 m$ справедливо соотношенuе

$$
[w(u, v)]_{k}=\overline{2^{l-1}} x_{k-2 m}^{1+\varepsilon_{1}} x_{k-2 m+2}^{\varepsilon_{2}} \cdots x_{k-4}^{\varepsilon_{m-1}} x_{k-2}^{\varepsilon_{m}}+h_{k}^{(w)}\left(x_{k-2 m}, \ldots, x_{k-1}\right),
$$

где $l$ - количество вхождений буквы $X_{2}$ в слово $w$,

$$
\varepsilon_{i}= \begin{cases}1, & \text { если на } i \text {-й позичии в } w \text { стоит } X_{2}, \\ 0 \quad \text { в противном случае, } & 1 \leqslant i \leqslant m,\end{cases}
$$

а многочлен $h_{k}^{(w)}$ не содержит монома $x_{k-2 m}^{1+\varepsilon_{1}} x_{k-2 m+2}^{\varepsilon_{2}} \cdots x_{k-4}^{\varepsilon_{m-1}} x_{k-2}^{\varepsilon_{m}}$.

Кроме того, если слово $w$ заканчивается буквой $X_{1}$, то многочлен $h_{k}^{(w)}$ не содерэит мономов вида $x_{k-2 m}^{\alpha_{1}} x_{k-2}^{\alpha_{2}} x_{k_{1}}^{\alpha_{3}} \cdots x_{k_{s}}^{\alpha_{s+2}}$ ни прикаких $s \geqslant 0, \alpha_{1}, \alpha_{2}>0$, $\alpha_{3}, \ldots, \alpha_{s+2} \geqslant 0$.

ДокАЗАТЕЛЬСТво. Проведем индукцию по длине слова $w$. Для слов длины 1 утверждение верно согласно определению таблиц $u$ и $v$. Пусть $|w|>1$ и слово $w$ заканчивается буквой $X_{1}$, т.е. имеет вид $\widetilde{w}\left(X_{1}, X_{2}\right) X_{1}$. Тогда при $k>2 m$

$$
[w(u, v)]_{k}=[\widetilde{w}(u, v)]_{k}+\alpha_{k-2}\left(x_{k-1}+[\widetilde{w}(u, v)]_{k-1}\right)+\left(x_{k-2}+[\widetilde{w}(u, v)]_{k-2}\right) .
$$

По предположению индукции $[\widetilde{w}(u, v)]_{k}+\alpha_{k-2}\left(x_{k-1}+[\widetilde{w}(u, v)]_{k-1}\right)$ является многочленом от переменных $x_{k-2 m+2}, \ldots, x_{k-1}$, поэтому не содержит мономов, указанных в условии леммы. Далее,

$$
[\widetilde{w}(u, v)]_{k-2}=\overline{2^{l-1}} x_{k-2 m}^{1+\varepsilon_{1}} x_{k-2 m+2}^{\varepsilon_{2}} \cdots x_{k-4}^{\varepsilon_{m-1}}+h_{k}^{(\widetilde{w})}\left(x_{k-2 m}, \ldots, x_{k-3}\right),
$$

т.е. не зависит от $x_{k-2}$ и не может содержать мономов вида $x_{k-2 m}^{\alpha_{1}} x_{k-2}^{\alpha_{2}} x_{k_{1}}^{\alpha_{3}} \cdots x_{k_{s}}^{\alpha_{s+2}}$ ни при каких $s \geqslant 0, \alpha_{1}, \alpha_{2}>0, \alpha_{3}, \ldots, \alpha_{s+2} \geqslant 0$. Значит, [w(u,v) $]_{k}$ есть сумма монома $\overline{2^{l-1}} x_{k-2 m}^{1+\varepsilon_{1}} x_{k-2 m+2}^{\varepsilon_{2}} \cdots x_{k-4}^{\varepsilon_{m-1}}$ и многочлена требуемого вида. Этот моном можно записать в виде $\overline{2^{l-1}} x_{k-2 m}^{1+\varepsilon_{1}} x_{k-2 m+2}^{\varepsilon_{2}} \cdots x_{k-4}^{\varepsilon_{m-1}} x_{k-2}^{\varepsilon_{m}}$, положив $\varepsilon_{m}=0$.

Если слово $w$ заканчивается буквой $X_{2}$, все рассуждения вполне аналогичны, поэтому такой случай рассматривать не будем. Лемма доказана.

TЕОРема 3. Полугруппа $F(\bar{\alpha})$ свободна.

ДокАЗАТЕЛЬСТво. Нужно показать, что для двух произвольных различных слов $w_{1}\left(X_{1}, X_{2}\right)$ и $w_{2}\left(X_{1}, X_{2}\right)$ из свободной полугруппы $\operatorname{FS}\left(X_{1}, X_{2}\right)$ их значения на $(u, v)$ не совпадают. В силу леммы 5 таблищы $u$ и $v$ имеют бесконечный порядок, т.е. достаточно рассмотреть только нетривиальные слова $w_{1}$ и $w_{2}$. Возможны два случая.

1) Слова $w_{1}$ и $w_{2}$ имеют различную длину. Предположим, что $\left|w_{1}\right|>\left|w_{2}\right|$ и $\left|w_{1}\right|=m$. Тогда по лемме 6 для $k>2 m$ имеем

$$
\left[w_{1}(u, v)\right]_{k}=\overline{2^{l-1}} x_{k-2 m}^{1+\varepsilon_{1}} x_{k-2 m+2}^{\varepsilon_{2}} \cdots x_{k-4}^{\varepsilon_{m-1}} x_{k-2}^{\varepsilon_{m}}+h_{k}^{\left(w_{1}\right)}\left(x_{k-2 m}, \ldots, x_{k-1}\right),
$$

где $l$ - количество вхождений $X_{2}$ в $w_{1}, \varepsilon_{i} \in\{0,1\}, 1 \leqslant i \leqslant m$, a $h_{k}^{\left(w_{1}\right)}$ не содержит монома $x_{k-2 m}^{1+\varepsilon_{1}} x_{k-2 m+2}^{\varepsilon_{2}} \cdots x_{k-4}^{\varepsilon_{m-1}} x_{k-2}^{\varepsilon_{m}}$, т.е. многочлен $\left[w_{1}(u, v)\right]_{k}$ явно зависит от неизвестного $x_{k-2 m}$. С другой стороны, по лемме $6\left[w_{2}(u, v)\right]_{k}$ от него не зависит. Следовательно, $\left[w_{1}(u, v)\right]_{k} \neq\left[w_{2}(u, v)\right]_{k}$, и значения $w_{1}$ и $w_{2}$ на $(u, v)$ различны. 
2) Слова $u$ и $v$ имеют одинаковую длину $m$. Можно считать, что их последние буквы различны, т.е. $w_{1}\left(X_{1}, X_{2}\right)=\widetilde{w}_{1}\left(X_{1}, X_{2}\right) X_{1}, w_{2}\left(X_{1}, X_{2}\right)=\widetilde{w}_{2}\left(X_{1}, X_{2}\right) X_{2}$. По лемме 6 при $k>2 m$ многочлен $\left[w_{1}(u, v)\right]_{k}$ не содержит мономов вида $x_{k-2 m}^{\alpha_{1}} x_{k-2}^{\alpha_{2}} x_{k_{1}}^{\alpha_{3}} \cdots x_{k_{s}}^{\alpha_{s+2}}$ ни при каких $s \geqslant 0, \alpha_{1}, \alpha_{2}>0, \alpha_{3}, \ldots, \alpha_{s+2} \geqslant 0$, а в многочлене $\left[w_{2}(u, v)\right]_{k}$ встречается моном $x_{k-2 m}^{1+\varepsilon_{1}} x_{k-2 m+2}^{\varepsilon_{2}} \cdots x_{k-4}^{\varepsilon_{m-1}} x_{k-2}$ с коэффициентом $\overline{2^{l-1}}\left(l-\right.$ число вхождений $X_{2}$ в $w_{2}$, а $\varepsilon_{i}=1$ или 0 в зависимости от того, стоит ли на $i$-й позиции в $w_{2}$ буква $X_{2}$ или нет, $1 \leqslant i \leqslant m-1)$. Следовательно, $\left[w_{1}(u, v)\right]_{k} \neq\left[w_{2}(u, v)\right]_{k}$, и значения $w_{1}$ и $w_{2}$ на кортеже $(u, v)$ снова различны. Теорема доказана.

Пусть теперь $p=2$. Таблицы $u$ и $v$ определим по последовательности $\bar{\alpha} \in \mathbb{Z}_{2}^{\infty}$ следующим образом:

$$
\begin{gathered}
u=\left[0,0,0, \alpha_{1} x_{3}+x_{1}, \alpha_{2} x_{4}+x_{2}, \ldots, \alpha_{k} x_{k+2}+x_{k}, \ldots\right], \\
v=\left[0,0,0, \alpha_{1} x_{3}+x_{1} x_{2}, \alpha_{2} x_{4}+x_{2} x_{3}, \ldots, \alpha_{k} x_{k+2}+x_{k} x_{k+1}, \ldots\right] .
\end{gathered}
$$

Как и раньше, обозначим через $F(\bar{\alpha})$ полугрупу, порожденную этими таблищами.

ТЕОРема 4. Полугруппа $F(\bar{\alpha})$ свободна.

ДокАЗАТЕЛЬСтво. Пусть $w\left(X_{1}, X_{2}\right)$ - нетривиальное слово из $\mathrm{FS}\left(X_{1}, X_{2}\right), m$ - его длина. Рассуждая, как в лемме 6 , можно показать, что при $k>3 m$ многочлен $[w(u, v)]_{k}$ содержит моном $x_{k-3 m} x_{k-3 m+1}^{\varepsilon_{1}} x_{k-3 m+4}^{\varepsilon_{2}} x_{k-3 m+7}^{\varepsilon_{3}} \cdots x_{k-5}^{\varepsilon_{m-1}} x_{k-2}^{\varepsilon_{m}}$, где $\varepsilon_{i}$ задаются формулой (9). При этом, если $w$ заканчивается буквой $X_{1}$, то $[w(u, v)]_{k}$ не содержит мономов, в которых одновременно встречаются неизвестные $x_{k-3 m}$ и $x_{k-2}$. Затем, как и в доказательстве теоремы 3 , рассмотрим два различньх нетривиальных слова $w_{1}$ и $w_{2}$ из $\operatorname{FS}\left(X_{1}, X_{2}\right)$. Если $\left|w_{1}\right|>\left|w_{2}\right|,\left|w_{1}\right|=m$, то при $k>3 m$ многочлен $\left[w_{1}(u, v)\right]_{k}$ зависит от переменной $x_{k-3 m}, \mathrm{a}\left[w_{2}(u, v)\right]_{k}$ от нее не зависит. Если же $\left|w_{1}\right|=\left|w_{2}\right|=m$, то, считая для определенности, что $w_{1}$ заканчивается буквой $X_{1}$, а $w_{2}$ - буквой $X_{2}$, получаем при $k>3 m$, что $\left[w_{1}(u, v)\right]_{k}$ содержит моном, в котором одновременно встречаются неизвестные $x_{k-3 m}$ и $x_{k-2}$, а в $\left[w_{2}(u, v)\right]_{k}$ таких мономов нет. Следовательно, для любых слов $w_{1}, w_{2} \in \mathrm{FS}\left(X_{1}, X_{2}\right)$ в таблищах $w_{1}(u, v)$ и $w_{2}(u, v)$ всегда найдутся различные координаты, т.е. значения $w_{1}$ и $w_{2}$ на кортеже $(u, v)$ не совпадают. Это и означает, что $F(\bar{\alpha})$ свободна. Теорема доказана.

Напомним, что автоматное преобразование $u \in A T_{n}$ называется конечно-автоматным, если его можно задать конечным автоматом. Произведение конечно-автоматных преобразований снова будет конечно-автоматным, т.е. все конечно-автоматныепреобразования образуют подполугруппу в $A T_{n}$, которую обозначим $F A T_{n}$. Подполугруппы из $F A T_{n}$ естественно назьвать полугруппами конечно-автоматных преобразований.

Если автоматное преобразование $u$ задано таблицей вида (1), то для произвольных $m \in \mathbb{N}$ и набора $\bar{a}=\left(a_{1}, a_{2}, \ldots, a_{m}\right) \in A^{m}$ можно определить таблищу

$$
\left[f_{m+1}\left(a_{1}, a_{2}, \ldots, a_{m}\right), \ldots, f_{m+k}\left(a_{1}, a_{2}, \ldots, a_{m}, x_{1}, x_{2}, \ldots, x_{k-1}\right), \ldots\right]
$$

которая также содержится в $A T_{n}$. Будем называть эту таблицу $(\bar{a}, m)$-остатком таблищы $u$. С помощью этого понятия легко формулируется критерий конечно-автоматности преобразований из $A T_{n}$.

Лемма 7. Автоматное преобразование из $A T_{n}$, которое задается таблиией и, является конечно-автоматным в том и только том случае, когда таблича и имеет лишь конечное число различных $(\bar{a}, m)$-остатков для всех $m \in \mathbb{N}, \bar{a} \in A^{m}$. 
ДокАЗАТЕЛьСтво. Для автоматных подстановок доказательство приведено в [15]. Общий случай ничем не отличается.

ТЕОремА 5. Полугруппа $F(\bar{\alpha})$ будет полугруппой конечно-автоматных преобразований тогда и только тогда, когда, начиная с некоторого места, последовательность $\bar{\alpha}$ является периодической.

ДокАЗАТЕЛЬСТво. Ограничимся случаем $p \neq 2$. При $p=2$ рассуждения вполне аналогичны.

Необходимость. Пусть последовательность $\bar{\alpha}$ непериодическая с предпериодом, т.е. среди последовательностей $\left(\alpha_{m}, \alpha_{m+1}, \ldots, \alpha_{m+k}, \ldots\right), m \geqslant 1$, бесконечно много различных. Покажем, что таблица

$$
u=\left[0,0, \alpha_{1} x_{2}+x_{1}, \alpha_{2} x_{3}+x_{2}, \ldots, \alpha_{k} x_{k+1}+x_{k}, \ldots\right]
$$

имеет бесконечно много различных остатков. Действительно, каждый ее остаток имеет вид $\left[\alpha, \alpha_{m} x_{1}+\beta, \alpha_{m+1} x_{2}+x_{1}, \ldots, \alpha_{m+k} x_{k+1}+x_{k}, \ldots\right]$ при некоторых $m \in \mathbb{N}$ и $\alpha, \beta \in \mathbb{Z}_{p}$. Поскольку среди последовательностей $\left(\alpha_{m}, \alpha_{m+1}, \ldots, \alpha_{m+k}, \ldots\right), m \geqslant 1$, бесконечно много различных, то и различных остатков у таблицы $u$ бесконечно много, т.е. по лемме 7 она не конечно-автоматна.

Достаточность. Если последовательность $\bar{\alpha}$ периодическая, начиная с некоторого места, т.е. имеет вид $\left(\alpha_{1}, \ldots, \alpha_{l}, \alpha_{l+1}, \ldots, \alpha_{l+m}, \alpha_{l+1}, \ldots, \alpha_{l+m}, \ldots\right)$ при каких-то $l \geqslant 0$, $m \in \mathbb{N}$, то таблищы $u, v$ имеют конечное число различных остатков. В самом деле, в этом случае любой остаток таблицы $u$ имеет вид

$$
\begin{aligned}
& {\left[\alpha, \alpha_{i} x_{1}+\beta, \alpha_{i+1} x_{2}+x_{1}, \ldots, \alpha_{l+m} x_{l+m-i+1}+x_{l+m-i},\right.} \\
& \quad \alpha_{l+1} x_{l+m-i+2}+x_{l+m-i+1}, \ldots, \alpha_{l+m} x_{l+2 m-i+1}+x_{l+2 m-i}, \\
& \left.\quad \alpha_{l+1} x_{l+2 m-i+2}+x_{l+2 m-i+1}, \ldots, \alpha_{l+m} x_{l+3 m-i+1}+x_{l+3 m-i}, \ldots\right]
\end{aligned}
$$

при некоторых $i \in\{1, \ldots, l+m\}$ и $\alpha, \beta \in \mathbb{Z}_{p}$. Отсюда получаем, что различных остатков у нее не больше чем $(l+m) p^{2}$. Аналогично, таблица $v$ имеет не больше чем $(l+m) p^{2}$ различных остатков. Следовательно, по лемме 7 полугруппа $F(\bar{\alpha})$ порождается конечно-автоматными преобразованиями, т.е. является полугрупшой конечно-автоматных преобразований. Теорема доказана.

Пусть $\chi=\left\{m_{1}, m_{2}, \ldots\right\}$ - бесконечная последовательность натуральных чисел такая, что $m_{k}<k$ при $k>1$.

ОПРЕДЕЛЕНИЕ 4. Таблицу $u=\left[f_{1}, f_{2}\left(x_{1}\right), \ldots, f_{k}\left(\bar{x}_{k-1}\right), \ldots\right] \in P_{\infty}$ назовем $\chi$ угольной, если существует номер $k_{0} \in \mathbb{N}$ такой, что при всех $k \geqslant k_{0}$ координаты $u$ имеют вид

$$
f_{k}\left(\bar{x}_{k-1}\right)= \begin{cases}\tilde{f}_{k}\left(x_{k-m_{k}+1}, \ldots, x_{k-1}\right)+x_{k-m_{k}}^{l_{k}}, l_{k} \geqslant 1, & \text { при } p>2, \\ \tilde{f}_{k}\left(x_{k-m_{k}+1}, \ldots, x_{k-1}\right)+x_{k-m_{k}-1} x_{k-m_{k}}^{\varepsilon_{k}}, \quad \varepsilon_{k} \in\{0,1\}, & \text { при } p=2,\end{cases}
$$

причем многочлен $\widetilde{f}_{k} \equiv 0$, если $m_{k}=1$.

$\chi$-треугольную таблицу будем назьвать $\chi$-диагональной, если при $k \geqslant k_{0}$ последовательность $\left\{m_{k}\right\}$ не возрастает.

Следующее утверждение является обобщением теорем 3 и 4 . 
Теорема 6. Если $p \neq 2$, то ұ-диагональные таблицы $и, v$, в которых первые $k_{0}-1$ координат произвольны, а последующ, опе опеделяются равенствами

$$
\begin{aligned}
& {[u]_{k}=\widetilde{f}_{k}\left(x_{k-m_{k}+1}, \ldots, x_{k-1}\right)+x_{k-m_{k}}, \quad k \geqslant k_{0},} \\
& {[v]_{k}=\widetilde{f}_{k}\left(x_{k-m_{k}+1}, \ldots, x_{k-1}\right)+x_{k-m_{k}}^{2}, \quad}
\end{aligned}
$$

порохдают свободную полугруппу.

Если $p=2$, то ұ-диагональные таблицы $u, v$, первые $k_{0}-1$ координат которых произвольны, а последующие определяются равенствами

$$
\begin{gathered}
{[u]_{k}=\widetilde{f}_{k}\left(x_{k-m_{k}+1}, \ldots, x_{k-1}\right)+x_{k-m_{k}-1},} \\
{[v]_{k}=\widetilde{f}_{k}\left(x_{k-m_{k}+1}, \ldots, x_{k-1}\right)+x_{k-m_{k}-1} x_{k-m_{k}}, \quad k \geqslant k_{0},}
\end{gathered}
$$

порохдают свободную полугруппу.

Поскольку доказательство этой теоремы отличается от доказательства теорем 3 и 4 только техническими деталями, мы его опускаем.

Автор выражает искреннюю благодарность профессору В. И. Сущанскому за постоянную помощь и внимание к работе.

\section{СПИСОК ЦИТИРОВАННОЙ ЛИТЕРАТУРЫ}

[1] Уфнаровский В. А. Комбинаторные и асимптотические методы в алгебре // Итоги науки и техн. Соврем. пробл. матем. Фундамент. направления. Т. 57. М.: ВИНИТИ, 1990. С. 5-177.

[2] Заровный В.П. Автоматные подстановки и сплетения групп // Докл. АН СССР. 1965. T. 160. №3. C. 562-565.

[3] Kaloujnine L.A., Beleckij P. M., Fejnberg V.Z. Kranzprodukte. Leipzig: Teubner, 1987.

[4] Wells C. Some applications of the wreath product construction // Amer. Math. Monthly. 1976. V. 83. P. 317-338.

[5] Алёшин С.В. Конечные автоматы и проблема Бернсайда о периодических группах // Матем. заметки. 1972. Т. 11. № 3. С. 319-328.

[6] Алёшин С. В. Свободная группа конечньх автоматов // Вестн. МГУ. Сер. 1. Матем., мех. 1983. № 4. C. 12-14.

[7] Tits J. Free subgroups in linear groups // J. Algebra. 1972. V. 20. P. 250-270.

[8] Epstein D. B. A. Almost all subgroups of a Lie group are free // J. Algebra. 1971. V. 19. P. 261-262.

[9] Dixon J. D. Most finitely generated permutation groups are free // Bull. London Math. Soc. 1990. V. 22. P. 222-226.

[10] Bhattacharjee M. The ubiquity of free subgroups in certain inverse limits of groups // J. Algebra. 1995. V. 172. P. 134-146.

[11] Кудрявцев В. Б., Алёшин С. В., Подколзин А. С. Введение в теорию автоматов. М.: Наука, 1985.

[12] Holland W.C. The characterization of generalized wreath product // J. Algebra. 1969. V. 13. P. $152-172$.

[13] Александрян Р. А., Мирзаханян Э. А. Общая топология. М.: Высшая школа, 1979.

[14] Серр ЖК.-П. Когомологии Галуа. М.: Мир, 1968.

[15] Сущанський В. І. Стандартні підгрупи групи ізометрій метричного простору цілих $p$-адичних чисел // Вісник КДУ. Сер. матем., мех. 1988. №30. С. 100-107.

Киевский национальный университет им. Т. Шевченко

Поступило 26.03 .96 